\title{
Three-Dimensional Modeling Technology of Virtual Plant in Digital Landscape
}

\author{
Pu Liu ${ }^{1,2,3 *}$, Yuan $\mathrm{Liu}^{2}$ and Xiaosheng $\mathrm{Xu}^{3}$ \\ ${ }^{1}$ Beijing Jiaotong University, No.3 Shangyuan Cun, Haidian District, Beijing, China \\ ${ }^{2}$ Jiangnan University, No.1800 Lihu Avenue, Binhu District, Wuxi, China \\ ${ }^{3}$ Wuhan University, No.299 Bayi Road, Wuchang district, Wuhan, China \\ ${ }^{*}$ Corresponding author
}

\begin{abstract}
Three-dimensional modeling is the foundation of graphic expression and virtual reality of digital landscape. This article explores key technologies, methods and applications of three-dimensional modeling of virtual plant in digital landscape; and classifies into manual modeling technology, program generation technology, software automatic generation technology, measurement-based reconstruction technology. Researching the visual simulation of landscape plant elements with threedimensional modeling theories of computer graphics, the main purpose is to provide three-dimensional expression for landscape design and landscape planning with theoretical guide, as well as to promote sustainable development of virtual plant modeling technology in the field of landscape architecture.
\end{abstract}

Keywords-landscape architecture; landscape planning; landscape design; computer-aided design; three-dimensional modeling; plant; tree; virtual reality

\section{INTRODUCTION}

Computer technology is not only changing the traditional design and plan method of landscape, but also changed the thinking mode of landscape. Computer applications in the field of landscape include data collecting, sharing, exchanging, extracting; spatial information analysis, landscape expression and evaluation, dynamic monitoring and management, plant selection, virtual landscape, remote design, etc. In such a wide range of applications, the three-dimensional model construction is a small aspect, but it is the basis of the threedimensional landscape. In the industry involving landscape architecture, the expression, design, virtual analysis and calculation of landscape are all rely on the construction of three-dimensional model.

In the field of three-dimensional modeling, the natural scenery simulation is the key subject. Virtual plant is the hot research area with cross disciplines of mathematics, computer graphics, botany and ecology. Plants are common elements in the landscape. Three-dimensional plant models are indispensable components in landscape models. The virtual plant in digital landscape is the computer simulation of structure forms or the growth processes of plant individuals or groups, based on plant ecological models, using virtual reality technologies. This paper researches the three-dimensional model of virtual plant, analyses its development, key technologies and methods in digital landscape, in order to better promote the three-dimensional model application in landscape practice and extend the research scope of virtual plant.

\section{RESEARCH BACKGROUND}

Virtual plants are realistic three-dimensional models of plant individuals or groups, reflecting the morphological structures and ecological processes of real plants. There are two main research directions of virtual plant: One is outward appearance simulation, based on three-dimensional simulation of computer graphics, emphasizing the lifelike visual performance, and exploring plant visualization research. The other one is the simulation of plant growth process, on the basis of plant physiology, cell biology, dynamics and mechanical discipline. Combining the two, a real plant can be simulated through the interaction of function models and structure models. However various species, complex structures, diversified forms of plants are quite difficult to model, storage or draw when simulating.

The study of virtual plant began in the 1960s. In 1968, American biologist A. Lindenrnayer proposed L-system which is the important theoretical basis for plant modeling and simulating. In the early 1970s, EELIA, the first plant modeling language based on L-system, was born, followed with the PFG and CPF. In 1984, R. Smith achieved computer plant image generation using the L-system. The L-system became one of the main methods of virtual plant modeling in early stage. Along with the particle system put forward by WT. Reeves, finite automation model by French CIRAD, CPFG by Calgary university, plant structure-function feedback mechanism model by GreenLab, consists of important methods of virtual plant modeling. With the upgrade of the computing and processing capacity, and the invention of related equipment of three-dimensional scanner and digitizer, more varied and efficient methods of three-dimensional plant modeling have been emerging. But specialized studies and reports of virtual plant modeling in the field of landscape are rare in the existing literature.

Virtual plant in the digital landscape are computer graphics models with visual performance. Objects can be expressed and in-depth analyzed, using the virtual alternatives to test and compare rather than the real one, so it is a simple, economic, fast and safe way with time saving and labor saving. Arbor, shrub and grass and other common plant elements construct the space, place, visual focus and vision field in the landscape, 
also provide with rich colors and varied material characteristics. They almost determine the space effect of the whole landscape, and are the most dynamic landscape elements. Virtual Plant model is an important component which directly affects the authenticity of the scene and the atmosphere of the natural environment when conducting virtual environment and landscape performance. Threedimensional plant modeling has also been the difficult part of landscape modeling. According to different purposes or different usages, different model building methods and techniques will be adopted. They are mainly divided into manual modeling technology, program generation technology, software automatic generation technology, and measurementbased reconstruction technology.

\section{Manual Modeling Technology}

\section{A. Wire-Frame Modeling Method}

From the perspective of computer graphics, threedimensional model is based on basic geometry elements of dot, line, plane, and volume. Whether simple or complex threedimensional model is constructed through geometric shape modelling. Wire frame model is also the basis of the surface model and solid model. The contour and edge is used to describe the geometric feature of the object. Once the vertexes and edges are set, the object is identified. so the lines can't be hidden automatically. This results the impression of intensive lines, which often make it tends to represent smaller size than the actual one. Using wire frame modeling, decomposition views and perspectives can be easily generated. Wireframe model illustrates the space effects therefore can be used to analyze the spatial relationships of plants. Three-dimensional wireframe model is mainly used as a kind of display mode in the beginning steps of the virtual plant modeling in digital landscape, to deliberate shapes or perform some specific figures.

\section{B. Surface Modeling Method}

Surface model builds the structure forms with its connecting edges and enclosed surfaces. Due to increasing surfaces and edges, it is more complicated than the wire frame model on the data. Surface model is on the basis of the wireframe model but increases the relevant surface feature information, edge information and edge connection direction information, etc. The surface model defines the boundary of the object, can hide lines, accept coloring, and cast shadows, etc., so produce realistic colored images, which almost meet all the requirements of designers for graphic expression of plants.

Plane mapping method is often used in the digital landscape. The principle is to set one piece of plane or two mutually perpendicular planes in the three-dimensional modeling software, and mapping the planes with plant facade texture images. A mapping image is a single plant facade photo with pure color background. When rendering, the pure background color is set transparent in order to get a penetration vision. The length-width ratio of the plane must keep the same as the ratio of the plant facade photo.
This method is suitable for medium long shot of plant graphic expression. The two-dimensional images of trees or other plants are directly introduced into three-dimensional scenes, so the computing speed is relatively fast with simple geometric forms. The disadvantage is that, due to depicting a tree just with the two-dimensional plane of the tree texture, the space distribution characteristic of the tree is lost. In addition to that, lighting effects cannot be expressed, so it is not ideal when close observing. If there are too many trees, the texture data is huge, so the demand for the display cache of the device is high. Currently there are two kind methods of this plane mapping modeling:

Billboard model method: the tree picture is posted on a two-dimensional plane, then place the plane into a threedimensional scene, and ensure that the plane always towards the observer, so that the tree looks stereo from the observer view sight. Billboard model can imitate very fine texture, keeping a very low number of polygon. But must consider the relative direction and position between each plane and the sight line of the observer.

Criss cross method: place two piece of plane with tree pictures in a perpendicular cross way in three-dimensional space. So it presents a fully stereo tree from all directions viewpoint. This method gets good effect without adjusting the plane direction.

\section{Solid Modeling Method}

Solid modeling constructs entities with the basic voxels. It set the boundaries as the same as the surface modeling, but the internal area is solid. Rotating, stretching, plastic transforming is always used in this method. The solid entities have the characteristics of thickness, quality, gravity center, etc. So solid models can be constructed and modified with more rich contents. The biggest advantage is that cutting and the boolean operation can be carried out. So it is especially suitable for deliberate work of landscape modelling. Most threedimensional modeling software provide various geometry and surface controlled methods to generate various entities. By changing the parameters, a variety of monomers or combinations can be produced.

The plants of simple structures and regular shapes can be easily simulated with geometry solids. Such as the trimmed garden hedges, neat evergreen shrubs, and the geometric characteristic trees. The trunks can be depicted with cylinders or cones. The crowns can be expressed with cylinders, spheres, or cones. Then, added the texture, color, light effect, the virtual scenes are beautiful and realistic. In order to express more carefully, bump maps or double-sided maps with more complex texture can be used.

The solid models of plant with unique postures and irregular shapes can be built with path lofting method. Lofting along straight or curved paths in a virtual three-dimensional space, branches of cylinders with changing diameter are generated. Then use some geometric capture operation to connect these branches and make appropriate modifications, a three-dimensional tree with diversified branches is created. 


\section{Mixed Method of Two and Three Dimensional Modeling}

The structure of a tree is divided into three majority part of the leaves, branches and trunk. The crown and the trunk are important parts of a tree from the viewpoint of morphology and taxonomy. Through the analysis of the crown and the trunk, preliminary identification is carried out on the types and characteristics of a tree. So trees and their spatial feature information can be described through setting the forms of crowns and trunks. Leaves, bark and other details of trees are difficult to handle for most systems, needing a lot of memory and render time. Therefore, in digital landscape, usually not model solid of each single blade. The combination of twodimensional and three-dimensional modeling method is a good way to achieve the desirable effect, with the two-dimensional surface of material texture of tree leaves and bark, and the three-dimensional geometry of tree trunks or frameworks. Alpha mapping planes are used to represent the characteristics of large area of blade clusters. For example, the layered canopy method is commonly practiced, using different sizes of map sheets to represent the canopy on a solid tree trunk. Models of this method show the depth, hierarchy, shadow of the canopy, and is especially suitable for conifers.

\section{Program Generation Technology}

The morphology plant models and the dynamic plant growth models are established with mathematical expressions and model parameters, which are summed up to abstract various morphological and physiological mechanism of the plant and its growth process. The models are mostly based on the fractal geometry. The basic parameters of angle, length, radius, rotation, number, ratio of tree branches are used to control the plant morphological structure and growth change. Through these parameters new branches are recursively generated, with random disturbance of branch parameters at nodes of new branches. The main plant simulation method of fractal geometry such as L-System, A-System, Iterated Function System, Diffusion Limited Aggregation, Partial System, etc.

\section{A. L-System Method}

L-System emphasizes at describing plant topology structure, and developed to various function extension versions including Context Sensitive L-systems, Stochastic Lsystems, Parametric L-systems, Open L-systems, time varying L-system, differential L-system. They are widely used because the description for the plant of strict fractal structure is very refined and easy to modify and expand. But the plant models generated are monotonous and rigid, unable to describe the dynamics growth process.

\section{B. A-System Method}

A-system controls branch growth forms with four parameters of different branch length and angle, and describes changes of branch growth with five kinds of growth speed mode. By setting random changes of branch according to certain statistical regularity, various plants can be vividly simulated. The basic plant shapes are divided into three categories from branches difference. A-system also simulate plant appearances under the influence of sunlight, gravity and wind through applying the attractor algorithm. Morphological structures of plants can be rapidly generated, but because the process depends on the designers understanding of plant geometry appearance, and not having time-related growth sequences, so this method cannot reflect potential forms.

\section{Iteration Function System Method}

IFS originates from the self-similarity research of fractal geometry. It complete plant simulation with very little data, showing advantages in image compression. It generates coloring graphics with shading gradient and rich texture. But IFS bears narrow scope of application with disadvantages of lacking understand and control of the plant topology structure, difficult to determine the compression ratio and transform parameters, and hard to tell the morphological difference of simulated plant.

\section{Diffusion-Limited Aggregation Method}

The principle is that a growing aggregation group forms after selecting a static particle as a seed on the plane grid and conducting repeat random collision around the seed. DLA model is mainly used to simulate almost all kinds of fractal growth and cohesion phenomenon. The growth process of plant root and the morphological structure of marine algae are suitable for the computer simulation based on DLA model. DLA is infrequently used for the simulation of static plant forms.

\section{E. Particle System Method}

Particle system use a lot of particles to describe irregular objects of nature. Each particle has random properties at any moment, such as size, shape, color, transparency, direction and speed of movement, etc., and goes through three life stages of generation, activity and death. The aggregation of particles alive at a certain moment makes up the model of particle system. It is not suitable for presenting the topology form of individual plant, but for macro simulation of forest and grass. Trees structured by particle system not have highly realistic appearance because obvious artificial trace, and the lighting effects can't be calculated according to the objective facts strictly.

\section{F. Stochastic Method}

Stochastic method mainly includes finite automation method, multi-scale plant topology MTG method, and dual scale automation method. It associates with plant growth mechanism closely, and overcome the problem that $\mathrm{L}$ system is difficult to simulate tall plants. The finite automation is also known as the reference axis technique, which describe development, growth, dormancy and death process of plants with markov chain theory and state transition graph. However, reference axis is not easy to describe some plant growth characteristics related to growth cycles, such as growth rhythm and delay, or need more state parameters to describe.

\section{G. Stepwise Growth System Method}

Stepwise growth system simulates trees' feature of simultaneously growing and correcting, and controls the tree 
modeling with several parameters starting from the overall geometry structure. It simplifies the model, then draws and renders trees according to the distance using multiresolution method, so provides results of overall high quality under this strategy. But sometimes the colors of trees in far distance are too simplified, which results into a blur drawing.

\section{H. Plant Growth Structure System Method}

This technique simulates the mode and process of plant growth, determining the branches by set the parameters of growth time, plant's age, growth speed of branches, and the number of bud, etc. And classifies the shapes by controlling the probability of growth, suspension, death, and bifurcation.

\section{Interaction-Based Plant Model Method}

The model contains a series geometry information and structural system components of plants. These hierarchy components manage the plant space structure and shape information. For example, the geometric shapes of branches are generated by the geometry component; Arranging and repeating the branches structure are conducted by the tissue component; Geometric transformation component is used for geometric transformation or deformation operation of plants and branches.

\section{Software Automatic Generation TeChNOLOgY}

The three-dimensional modeling goes further automatically with the application of some software. These software can be divided into two classes. One kind is pure graphics software, mainly simulating plant shapes and external appearances, including Tree Factory, Speed Tree, Tree Storm, Onyx Tree, Forest Pro, and Xfrog, etc. The Tree Factory, Speed Tree, and Tree Storm use the technology of mixing three-dimensional and two-dimensional method. This kind of software generally enclose trees library, trees animation, trees designer etc. So users can select parameters to generate trees and animations automatically. The other kind of software are based on the structure model and function model, considering the structure and function of trees as much as possible. This kind of software include Amap, APSIM, GePSi, OWSimu, GPSF, SPASS, Cotton Plus, and Virtual the Plants, etc. Amap software is based on the pure three-dimensional model. Manual modeling technology are often mixed with software automatic generation technology in the procedure of landscape modeling.

\section{Measurement-Based Reconstruction TECHNOLOGY}

Models of this technique need to acquire the real plants spatial data with the application of three-dimensional digitizer, and then directly input the data into the computer, finally realize the three-dimensional simulation of real plants with the specially written program which calls the spatial data. The simulation effect is closely related to the precision of the digitizer instrument which measure plant spatial data. The measurement-based reconstruction model is able to accurately reproduce the morphological structure of static plant, directly using a lot of measurement data, but can't reflect the dynamic regulation of plant structure or forecast the dynamic change of plant structure.

\section{DISCUSSION AND CONCLUSION}

Landscape model construction refers to a great number of factors. This paper only considers the plant, the basic element of landscape, as the research object to explore the threedimensional modeling technologies, methods and applications. The theoretical methods and techniques can guide virtual plant and other objects modeling in digital landscape practice. while there are still many work need to be further studied.

How to optimize plant models and build plant models simply and quickly. How to better simulate the real object of natural plant, at the same time realize the information compression, reduce the information storage volume and transmission time. These questions in digital landscape are very important in practical application, and have always been targets for model builders. Model validity test is a significant problem too. Establishing and developing a set of general, quantifiable evaluation method, will promote the study of effectiveness of modeling.

With the progress of science and technology, many new methods and techniques are constantly emerging, which are necessary to research and develop. More and better threedimensional model building methods will be summarized in the practical application, to guide the modeling of virtual plant in digital landscape.

\section{ACKNOWLEDGMENT}

Jiangnan University Independent Scientific Research Program Youth fund JUSRP11556

\section{REFERENCES}

[1] P. Prusinkiewicz, "Modeling of spatial structure and development of plants: a review,” Scientia Horticulturae, 1998, vol. 74, pp. 113-149.

[2] D. Liu, Y. P. Zhu, and H. L. Liu, "Research progress on 3D plant visualization,” Journal of Agricultural Science \& Technology, 2015. pp. 27-38.

[3] T. Honjo, K. Saito, and Y. Kumagai, "A Study of the Application of Plant Modeling and Its Visualization on Landscape Prediction,” Journal of the Japanese Institute of Landscape Architects, 1991, vol. 55, pp. 301306 .

[4] Q. L. Zhang and M. Y.Pang, "A Survey of Modeling and Rendering Trees," Technologies for E-Learning and Digital Entertainment. Springer Berlin Heidelberg, 2008: pp. 757-764.

[5] L. U. Sheng lian and X. Y. Guo, "Research on Techniques for Accurate Modeling and Rendering 3D Plant Leaf,” Journal of Image \& Graphics, 2009. pp. 56-64.

[6] X. Gu and L. Xu, "3D reconstruction and visualization of plant leaves," Proceedings of SPIE - The International Society for Optical Engineering, 2015, pp. 443.

[7] P. Liu and Z. H. Peng "Three-Dimensional Modeling of Virtual City: Taking MultiGenCreator for Example,” Advanced Materials Research, 2013, vol. 711, pp. 617-622.

[8] T. Zhu, F. Tian, and Y. Zhou, "Plant Modeling Based on 3D Reconstruction and Its Application in Digital Museum,” Ijvr, 2008, vol. 7, pp. 81-88.

[9] P. Liu and Z. H. Peng, "Implementation Technologies and Procedure of the Real-time Interactive Roaming System of Virtual City,” Open Automation \& Control Systems Journal, 2013, vol. 5, pp. 21-29 